\title{
Comprehensive Evaluation of the Utility of 20 Endogenous Molecules as Biomarkers of OATP1B Inhibition Compared with Rosuvastatin and Coproporphyrin [s]
}

\author{
Shelby Barnett, Kayode Ogungbenro, Karelle Ménochet, Hong Shen, W. Griffith Humphreys, \\ and Aleksandra Galetin
}

Centre for Applied Pharmacokinetic Research, School of Health Sciences, University of Manchester, United Kingdom (S.B., K.O., A.G.); Non-Clinical PKPD, UCB, Slough, United Kingdom (K.M.); and Pharmaceutical Candidate Optimization, Bristol-Myers Squibb, Princeton, New Jersey (H.S., W.G.H.)

Received August 20, 2018; accepted October 9, 2018

\begin{abstract}
Endogenous biomarkers can be clinically relevant tools for the assessment of transporter function in vivo and corresponding drug-drug interactions (DDIs). The aim of this study was to perform systematic evaluation of plasma data obtained for 20 endogenous molecules in the same healthy subjects $(n=$ 8-12) in the absence and presence of organic anion transporting polypeptide (OATP) inhibitor rifampicin $(600 \mathrm{mg}$, single dose). The extent of rifampicin DDI magnitude [the ratio of the plasma concentration-time area under the curve (AUCR)], estimated fraction transported $\left(\mathrm{f}_{\mathrm{T}}\right)$, and baseline variability was compared across the biomarkers and relative to rosuvastatin and coproporphyrin I (CPI). Out of the 20 biomarkers investigated tetradecanedioate (TDA), hexadecanedioate (HDA), glycocholic acid, glycodeoxycholic acid (GDCA), taurodeoxycholic acid (TDCA), and coproporphyrin III (CPIII) showed the high AUCR (2.1-8.5) and $\mathrm{f}_{\mathrm{T}}(0.5-0.76)$ values, indicative of substantial OATP1Bmediated transport. A significant positive correlation was observed
\end{abstract}

between the individual GDCA and TDCAAUCRs and the magnitude of rosuvastatin-rifampicin interaction. The CPI and CPIII AUCRs were significantly correlated, but no clear trend was established with the rosuvastatin AUCR. Moderate interindividual variability (15\%-62\%) in baseline exposure and AUCR was observed for TDA, HDA, and CPIII. In contrast, bile acids demonstrated high interindividual variability $(69 \%-113 \%)$ and significant decreases in baseline plasma concentrations during the first 4 hours. This comprehensive analysis in the same individuals confirms that none of the biomarkers supersede $\mathrm{CPI}$ in the evaluation of OATP1Bmediated DDI risk. Monitoring of CPI and GDCATTDCA may be beneficial for dual OATP1B/sodium-taurocholate cotransporting polypeptide inhibitors with consideration of challenges associated with large inter- and intraindividual variability observed for bile acids. Benefit of monitoring combined biomarkers (CPI, one bile acid and one fatty acid) needs to be confirmed with larger data sets and against multiple OATP1B clinical probes and perpetrators.

\section{Introduction}

Drug-drug interaction (DDI) studies are required to determine the safety of a new molecular entity in drug development (Food and Drug Administration, 2017). In recent years, the impact of transporter and transporter-metabolism DDIs on systemic and tissue exposure has become increasingly evident (Gertz et al., 2013; Prueksaritanont et al., 2014; Hibma et al., 2016; Snoeys et al., 2016; Galetin et al., 2017). Several therapeutic classes rely on organic anion transporting polypeptide (OATP) 1B for uptake into the liver, e.g., statins and hepatitis $\mathrm{C}$ antivirals (Giacomini et al., 2010). Given that these are likely co-medications, it is crucial to evaluate the

S.B. is supported by a Ph.D. studentship from the Biotechnology and Biological Sciences Research Council, UK (BB/L502376/1) and UCB, UK.

https://doi.org/10.1124/jpet.118.253062.

S This article has supplemental material available at jpet.aspetjournals.org. potential of new molecular entities as OATP1B inhibitors. Basic in vitro-in vivo extrapolation of transporter inhibition data using static models to evaluate initial DDI risk can often produce false positive predictions (Vaidyanathan et al., 2016). To refine the assessment of transporter and/or transportermetabolism DDI risk in a mechanistic manner, physiologically based pharmacokinetic (PK) models are increasingly used (Gertz et al., 2013; Tsamandouras et al., 2015; Snoeys et al., 2016; Galetin et al., 2017; Guo et al., 2018). However, the confidence in physiologically based PK predictions of often complex transporter-mediated DDIs is still low compared with successes observed in the case of metabolic interactions (Jones et al., 2015; Food and Drug Administration, 2017).

Endogenous biomarkers are envisaged as clinically relevant tools for the early evaluation of OATP1B-mediated DDIs. Over recent years, increased numbers of DDI studies have monitored changes in the exposure of endogenous

ABBREVIATIONS: AUC, area under the curve; $\mathrm{AUC}_{0-24}$, area under the curve from time 0 to 24 hours; $\mathrm{AUCR}$, ratio of the area under the curve; $\mathrm{BA}$, bile acid; CPI, coproporphyrin I; CPIII, coproporphyrin III; DCA, deoxycholic acid; DDI, drug-drug interaction; EBI, endogenous biomarker interaction; $\mathrm{f}_{\mathrm{T}}$, fraction transported; GCA, glycocholic acid; GCDCA-S, sulfate conjugate of glycochenodeoxycholic acid; GDCA, glycodeoxycholic acid; HDA, hexadecanedioate; LCA, lithocholic acid; OATP, organic anion transporting polypeptide; PK, pharmacokinetic; $T_{0}$, plasma concentration at time zero; TDA, tetradecanedioate; TDCA, taurodeoxycholic acid. 
biomarkers in the presence of OATP1B inhibitors; these studies have been done in preclinical species (Chu et al., 2015; Watanabe et al., 2015; Shen et al., 2016; Thakare et al., 2017) and in humans (Lai et al., 2016; Shen et al., 2017, 2018; Takehara et al., 2017, 2018; Kunze et al., 2018; Liu et al., 2018).

Selectivity, sensitivity, and minimal variability in the baseline condition need to be considered for evaluation of endogenous molecules as potential clinical biomarkers of transporter function in vivo (Chu et al., 2017; Chu et al., 2018; Müller et al., 2018; Rodrigues et al., 2018). However, interpretation of endogenous biomarker interaction (EBI) data can be challenging due to other occurring physiologic processes usually not considered with clinical probes, such as synthesis, postprandial release, and diurnal rhythm (Rodrigues et al., 2018). Additionally, enterohepatic recirculation of some biomarkers [e.g., bile acids (BAs)] via bile salt export pump and organic solute transporters $\alpha$ and $\beta$ (Mok et al., 1977; Dawson, 2011; Hegade et al., 2017) may contribute to false negative interactions if the new molecular entity is also an inhibitor of this reabsorption mechanism. Guidelines on clinical study design and data interpretation for the use of endogenous molecules to evaluate transporter function in vivo are still evolving (Chu et al., 2018; Rodrigues et al., 2018). The majority of the currently reported data for endogenous OATP1B biomarkers come from screening studies in healthy subjects following a single dose of rifampicin (Shen et al., 2017; Takehara et al., 2017, 2018). Recently, a number of studies have monitored coproporphyrin I (CPI) following the administration of weak and moderate OATP1B inhibitors (Kunze et al., 2018; Liu et al., 2018; Shen et al., 2018). The reported studies focused on comparison of the plasma concentration-time area under the curve (AUC) or mean magnitude of DDI [ratio of the AUC (AUCR)] between one of the clinical probes (rosuvastatin, atorvastatin, and pravastatin) and CPI (Lai et al., 2016; Yee et al., 2016; Takehara et al., 2017, 2018; Kunze et al., 2018; Liu et al., 2018). In addition, nonlinear mixed-effects modeling of CPI plasma and urine data in the presence/absence of rifampicin was recently performed (Barnett et al., 2018), highlighting the selectivity and sensitivity of this endogenous molecule to monitor OATP1B transporter function in vivo. This analysis also reported low inter- and intraindividual variability of CPI and high fraction transported (76\%), attributed to OATP1B1/1B3 (Barnett et al., 2018).

The aim of this study was to expand on the previous analysis by Shen et al. (2017) and compare the magnitude of rifampicin interaction observed for 20 endogenous molecules using the data reported in the same healthy subjects as for the clinical probe rosuvastatin and CPI (the most established OATP1B endogenous biomarker). The analysis focused on estimation of the fraction transported $\left(f_{\mathrm{T}}\right)$ for each individual endogenous molecule investigated, assessment of biomarker baseline variability, reproducibility of EBI, and effect of food on biomarker exposure and data interpretation. In addition, multivariate regression analysis was performed to explore the utility of monitoring multiple biomarkers to support early identification of OATP1B-mediated DDI risk.

\section{Materials and Methods}

Clinical Data. Individual concentration-time data were obtained from a study reported previously in 12 healthy male subjects, all wild type for SLCO1B1 c.521T >C (Lai et al., 2016). The study was split into three occasions with a 7-day washout period between each occasion. In each phase, plasma and urine samples were collected predose [plasma concentration at time zero $\left.\left(T_{0}\right)\right]$ and at multiple time points up to 24 hours; subjects were fasted overnight and until 4 hours following administration of drug. A single $600 \mathrm{mg}$ oral dose of rifampicin and a single $5 \mathrm{mg}$ oral dose of rosuvastatin were administered to the volunteers in occasions 1 and 2 , respectively. On the third occasion the same respective doses of rifampicin and rosuvastatin were coadministered to healthy subjects (rosuvastatin/rifampicin occasion). Samples were analyzed for rifampicin, rosuvastatin, and 21 endogenous molecules as detailed in Lai et al. (2016) [CPI, coproporphyrin III (CPIII), and bilirubins] and Shen et al. (2017) (remaining biomarkers). Although 12 subjects participated in the trial, individual biomarker data were measured in 6-12 subjects depending on the phase of the trial, as detailed in Shen et al. (2017). In addition to rosuvastatin and CPI, endogenous molecules considered for analysis were tetradecanedioate (TDA), hexadecanedioate (HDA), glycocholic acid (GCA), glycodeoxycholic acid (GDCA), taurodeoxycholic acid (TDCA), dehydroepiandrosterone sulfate, deoxycholic acid (DCA), cholic acid, chenodeoxycholic acid, glycochenodeoxycholic acid, glycoursodeoxycholic acid, lithocholic acid (LCA), taurocholic acid, taurochenodeoxycholic acid, taurohyodeoxycholic acid/tauroursodeoxycholic acid, ursodeoxycholic acid/hyodeoxycholic acid, unconjugated bilirubin, total bilirubin, direct bilirubin, and CPIII.

Calculation of the Plasma Area under the Curve. The workflow of endogenous biomarker data analysis is illustrated in Fig. 1. The AUCs were calculated using the trapezoidal method in MATLAB 2016a (MathWorks, Natick, MA). Individual AUCs were calculated for all endogenous molecules investigated and rosuvastatin in the presence and absence of rifampicin. Subsequently, individual AUCRs were calculated (eq. 1) for both interaction occasions (rifampicin alone or rosuvastatin/rifampicin), where the AUC of each endogenous biomarker in the presence of rosuvastatin alone was used as the control. AUCRs were reported as the arithmetic mean and the interindividual variability for the AUCR of each biomarker in both occasions was assessed by calculating the CV percentage. Data for each individual biomarker are listed in Supplemental Table 1.

$$
\mathrm{AUCR}=\frac{\mathrm{AUC}(+ \text { rifampicin })}{\mathrm{AUC}(\text { control })}
$$

where AUC (control) and AUC (+ rifampicin) represent the AUC in the absence and presence of rifampicin, respectively.

Calculation of Fraction Transported. The fraction eliminated by transporters $\left(f_{T}\right)$ for each individual was calculated based on changes in biomarker AUC in the presence of a single $600 \mathrm{mg}$ dose of prototypical OATP inhibitor rifampicin, as shown in eq. 2 and defined previously for CPI and rosuvastatin (Barnett et al., 2018). The assumption was that changes in the systemic exposure of the endogenous molecule in the presence of rifampicin are reflective of the contribution of OATP1B, assuming that passive uptake is minimal relative to the active process and that OATP1B-mediated transport is a rate-determining step in the hepatic disposition of a potential biomarker. However, knowledge gaps with respect to in vitro transporter affinity, contribution of OATPs and/or sodium-taurocholate cotransporting polypeptides (NTCP), and contribution of passive process still exist for a number of endogenous molecules (summaries of the current data are shown in Supplemental Fig. 1 and Supplemental Table 2). In addition, it was assumed that administration of rifampicin or rosuvastatin had no effect on the synthesis of any of the endogenous molecules investigated.

$$
\mathrm{f}_{\mathrm{T}}=1-\frac{\text { AUC }(\text { control })}{\operatorname{AUC}(+ \text { rifampicin })}
$$

The $f_{T}$ for each probe was calculated for each individual subject, alongside the mean $f_{T}$ and the associated interindividual variability (data for each biomarker are listed in Supplemental Table 3). In cases 


\section{Preliminary analysis of 20 endogenous molecules}

- Calculation of AUCR and $\mathrm{f}_{\mathrm{T}}$ on two rifampicin occasions*

- Inter- and intra-individual variability of plasma $\mathrm{T}_{0}$

\section{Selection criteria for endogenous molecules}

- Weighted $A U C R>2$ and $\mathrm{f}_{\mathrm{T}}>50 \%$

- Consideration of plasma $T_{0}$ variability

\section{Further analysis for selected endogenous biomarkers (EB) $(6 / 20)$}

\author{
- Correlation between EB AUCR and rosuvastatin AUCR*, CPI \\ AUCR and rifampicin unbound $\mathrm{C}_{\max }$ \\ - Correlation between combined EB AUCR and rosuvastatin AUCR \\ - $\mathrm{EB}$ baseline plasma $\mathrm{AUC}_{0-24 \mathrm{~h}}$ stability \\ - Reproducibility of interaction \\ - Sensitivity of EB to inhibitor PK - case example
}

\section{Final selection of EBs suitable for the assessment of OATP1B interactions alongside CPI}

Fig. 1. Workflow of the data analysis for the selection of OATP1B endogenous biomarker; * denotes analysis based on the previous work reported for CPI (Barnett et al., 2018). where interaction AUC was lower than baseline AUC (due to high interoccasion variability in plasma exposure and unstable baseline), $\mathrm{f}_{\mathrm{T}}$ could not be calculated and those individual subjects were removed from the $f_{\mathrm{T}}$ analysis.

Assessment of Variability in $T_{0}$ Plasma Concentrations of Endogenous Biomarkers. Variability of baseline $\left(T_{0}\right)$ plasma concentrations was assessed for each endogenous molecule, as done previously for CPI (Barnett et al., 2018). For each occasion, the mean pre-rifampicin dose $T_{0}$ plasma concentrations of an endogenous biomarker and the corresponding interindividual variability were calculated (data for each individual biomarker are listed in Supplemental Table 4). In addition, variability in intraindividual baseline concentrations was assessed by calculating the CV across the three DDI phases (the range for each biomarker is listed in Supplemental Table 4).

Selection of Biomarkers for Subsequent Analysis. Following the preliminary analysis, a subset of biomarkers was selected for further investigation. Given that there were two inhibition occasions and a different number of subjects in each occasion, endogenous molecules were selected for subsequent analysis provided that the weighted mean of the AUCR (in the presence of rifampicin) and $f_{\mathrm{T}}$ were above 2 -fold and $50 \%$, respectively (Fig. 1). This selection was further refined by taking into consideration the variability in $T_{0}$ plasma concentrations. Variability was categorized as low, moderate, and high for $\mathrm{CVs}$ of $<25 \%,<50 \%$, and $>50 \%$, respectively.

Multiple regressions analyses were performed in $\mathrm{R}$ ( $\mathrm{R}$ Core Team, Vienna, Austria, version 3.4.1) to determine any correlations between the AUCR of selected biomarkers and either the rosuvastatin AUCR, CPI AUCR, or unbound rifampicin $C_{\max }\left(C_{\max , \mathrm{u}}\right)$ data obtained in the same individual (Supplemental Figs. 2 and 3). These analyses were performed for both EBI occasions using the respective endogenous biomarker AUCR data. Since rosuvastatin DDI were only reported on one occasion, the endogenous biomarker AUCRs from two occasions were compared with the same rosuvastatin AUCR data. Significant linear correlations of EBI and rosuvastatin AUCR, CPI AUCR, or rifampicin $C_{\max , \mathrm{u}}$ were identified as having a nonzero slope $(P<0.05)$. In addition, multivariate regression analysis was performed to assess whether combinations of biomarkers would recapitulate the extent of rifampicin interaction observed with a clinical probe rosuvastatin. Due to the subject size, combinations were limited to a maximum of three biomarkers. In cases when AUCR values of individual biomarkers were highly correlated (e.g., for CPI and CPIII, TDA and HDA, and GDCA and TDCA), these biomarkers were not investigated in combination in multivariate analysis. In addition to biomarkers, rifampicin $C_{\text {max,u }}$ was also considered in multiple regressions analysis. To assess the best combinations of biomarkers that could recapitulate rosuvastatin AUCR, the adjusted $R^{2}$ and $P$ values (nonzero slope, $P<0.05$ ) were used.

In addition, stability in baseline AUC and reproducibility of biomarker AUCR in the same individuals on two separate occasions (rifampicin and rosuvastatin/rifampicin occasions) were investigated. For the assessment of the reproducibility of biomarker interaction with rifampicin in the same individuals, boundaries in AUCR analogous to bioequivalence $(80 \%-125 \%)$ were set. Finally, the relationship between perpetrator (rifampicin) $\mathrm{PK}$ and the plasma profile of the biomarker was investigated at each time point.

Whenever possible, aspects of the analysis highlighted previously were also performed with any recent literature reported EBI data with these endogenous molecules. For example, a separate analysis was conducted for CPI using recently reported clinical EBI data to assess the variability of its plasma baseline AUC across several studies (data collated from 91 subjects in total). Due to limited availability of individual CPI data reported in those studies, the weighted baseline AUC from time 0 to 24 hours $\left(\mathrm{AUC}_{0-24}\right)$ and between-study variability were calculated from the mean CPI AUC data reported in five separate studies (Lai et al., 2016; Kunze et al., 2018; Liu et al., 2018; Shen et al., 2018; Takehara et al., 2018). Given that plasma data were only collected for 6 hours in Kunze et al. (2018), the AUC for CPI was 
extrapolated to 24 hours assuming that plasma concentrations remained stable between 6 and 24 hours. In the case of bile acids, reproducibility of rifampicin EBI reported in two clinical studies in Japanese volunteers was assessed (Takehara et al., 2017, 2018). In both interaction studies a $600 \mathrm{mg}$ oral dose of rifampicin was administered; therefore, the AUCR and AUC of the biomarker in the baseline condition were compared between studies.

Statistical Analysis. All statistical analyses were performed in GraphPad Prism (GraphPad Software, La Jolla California, version 7). For all endogenous biomarkers, the baseline $\left(T_{0}\right)$, AUCR, and $f_{T}$ parameters obtained in each occasion were checked for normal distribution using a Shapiro-Wilk normality test, and then appropriate parametric or nonparametric statistical tests were performed. For paired statistical analysis, subjects with incomplete data sets were removed (Supplemental Table 5). Therefore, a full set of interaction data was available for CPI and CPIII in 10 subjects, whereas data for the remaining biomarkers were only available in six out of 10 subjects. For all statistical tests a confidence interval of $95 \%$ was set $(P<0.05)$. To determine any significant differences in biomarker AUCR between rifampicin and rosuvastatin/rifampicin inhibition occasions either a Wilcoxon test (non-normal distribution) or a paired Student's $t$ test (normal distribution) was performed. Due to differences in number of $f_{T}$ values for some biomarkers/occasions when these values could not be calculated, an unpaired Student's $t$ test was performed to determine significant differences in $\mathrm{f}_{\mathrm{T}}$ between inhibition occasions. To determine any significant differences in biomarker $T_{0}$ plasma concentrations across three occasions either a Friedman test with Dunn's multiple comparisons correction (non-normal distribution) or repeated measures one-way analysis of variance ANOVA, (normal distribution) was performed.

Additional statistical analysis was conducted only for the endogenous molecules investigated in the selected subset. The stability of the baseline plasma profile (rosuvastatin occasion) for each biomarker in the selected subset was assessed through comparison of each plasma concentration (0.5-24 hours) to $T_{0}$, significant differences were determined using a Friedman test $(P<0.05)$. In the rosuvastatin occasion, one subject failed to attend, thus for the analysis of CPI and CPIII the data for 11 subjects were used, whereas for the remaining biomarkers the plasma data from seven subjects were available. To determine the sensitivity of the endogenous molecule, the time point when rifampicin causes a significant increase in biomarker plasma concentration $(P<0.05)$ was assessed. A repeated measures two-way ANOVA was performed for the selected biomarker subset, using only subjects with full interaction data (10 out of 12 subjects for CPI and CPIII and six out of 12 subjects for the remaining biomarkers). The analysis involved comparison of the plasma concentration-time profiles for different phases of the EBI study for each time point.

\section{Results}

Preliminary Analysis of Endogenous Molecules. The mean AUCR of 20 endogenous molecules in the presence of a single dose rifampicin was assessed on two separate occasions (individual values are listed in Supplemental Table 1). Out of 20 biomarkers investigated, 10 showed weighted mean of AUCR $>2$ on both inhibition occasions; namely, TDA, HDA, DCA, GCA, GDCA, glycoursodeoxycholic acid, taurocholic acid, TDCA, taurochenodeoxycholic acid, and CPIII. Although the AUCRs for the majority of endogenous molecules showed no significant differences between occasions $(P>0.05)$, there were some exceptions. DCA showed inconsistency in the magnitude of EBI, since a strong interaction with rifampicin $($ AUCR $=12.7)$ with the highest degree of variability $(196 \%)$ was only observed in the rifampicin-alone occasion. In contrast, no clinically significant EBI was noted for DCA during the rosuvastatin/rifampicin occasion $(\mathrm{AUCR}=1.1)$. In the case of LCA and dehydroepiandrosterone sulfate, no clinically significant interaction was observed in the presence of rifampicin, suggesting that their transport is not facilitated by OATPs. The range of individual AUCR values for the selected subset of endogenous molecules on both inhibition occasions is shown in Fig. 2. CPI and rosuvastatin (mean AUCRs of 4.3 and 5.2 , respectively) were used as references for the assessment of EBI magnitude of the remaining selected biomarkers. Similar to CPI, TDA, HDA, and GCA showed low interindividual variability in AUCR (15\%-32\%). However, the magnitude of interaction observed for TDA (AUCR $=2.8$ ), HDA (2.4), and GCA (2.1) was lower than observed for both CPI and rosuvastatin. In contrast, GDCA and TDCA showed the greatest interaction with weighted mean AUCRs of 8.5 and 5.1, respectively. However, the magnitude of EBI was highly variable between rifampicin inhibition occasions (Fig. 2). In contrast to CPI, the mean AUCR values of the selected biomarkers for each rifampicin inhibition occasion were not in good agreement with the mean AUCR for rosuvastatin.

For the 20 biomarkers investigated, a wide range in the $f_{T}$ values was calculated, spanning from 0.04 to 0.79 for LCA and GDCA, respectively (details are listed in Supplemental Table 3). Ten out of 20 of the endogenous molecules demonstrated a weighted mean $f_{T}>50 \%$. However, the $f_{T}$ for chenodeoxycholic acid, LCA, and ursodeoxycholic acid/hyodeoxycholic acid could only be calculated in a maximum of three subjects following inhibition with rifampicin in either of the occasions (due to either high variability and/or weak interaction) and were therefore excluded from further analysis. Both GDCA and TDCA showed high weighted mean $\mathrm{f}_{\mathrm{T}}$ in the rifampicin inhibition study (0.79 and 0.71 , respectively), comparable to rosuvastatin (0.79) and CPI (0.76) (Barnett et al., 2018) (Fig. 3). In contrast, these values could not be reproduced in the rosuvastatin/rifampicin occasion, which resulted in inconsistent and generally lower mean $\mathrm{f}_{\mathrm{T}}$ in this phase (0.64 and 0.46 for GDCA and TDCA, respectively). Although the mean $\mathrm{f}_{\mathrm{T}}$ for TDA (0.64), HDA (0.58), and GCA (0.5) was lower than observed for rosuvastatin and CPI, it was $\geq 50 \%$ on both occasions, with low interindividual variability $(<28 \%)$ (Fig. 3). Based primarily on their AUCR and $\mathrm{f}_{\mathrm{T}}$, and to some extent the stability in $T_{0}$, biomarkers TDA, HDA, GCA, GDCA, TDCA, and CPIII were selected for further evaluation and comparison with rosuvastatin and CPI.

Correlation of Endogenous Biomarker AUCR with Rosuvastatin and CPI. Multiple regressions analyses were performed to identify any correlations between the extent of $\mathrm{EBI}$ and rosuvastatin AUCR, CPI AUCR, and rifampicin $C_{\text {max,u }}$ (Fig. 4; Supplemental Figs. 2-8). Only two out of six of the endogenous molecules (GDCA and TDCA) showed a significant positive correlation with rosuvastatin AUCR (Fig. 4). These biomarkers also showed the greatest mean AUCR following administration with rifampicin of 13.7- and 7.4-fold, respectively. In contrast, these trends were not captured in the rosuvastatin/rifampicin inhibition occasion, highlighting the lack of EBI reproducibility observed for GDCA and TDCA in this subject cohort (Table 1). No significant correlation was observed between the AUCR for the remaining biomarkers relative to rosuvastatin in either of the inhibition occasions (Fig. 4; Supplemental Fig. 4). CPIII was the only endogenous molecule that showed a significant positive correlation with CPI AUCR data (Fig. 4), whereas no direct relationship could be established between the EBI magnitude 
TDA
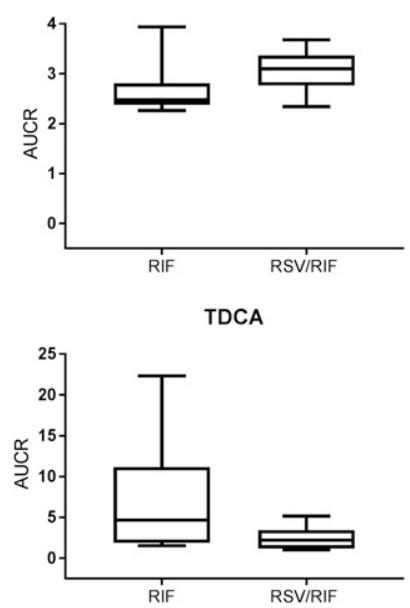

HDA
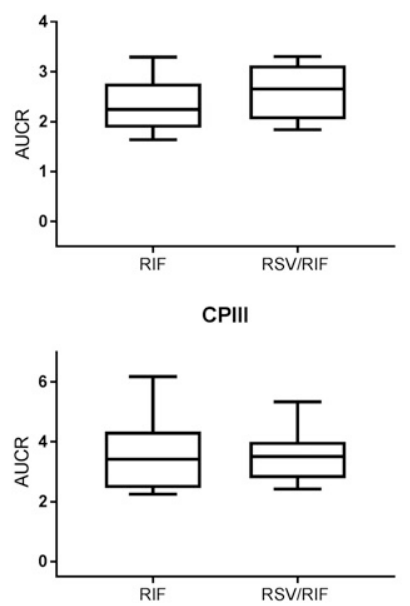

GCA
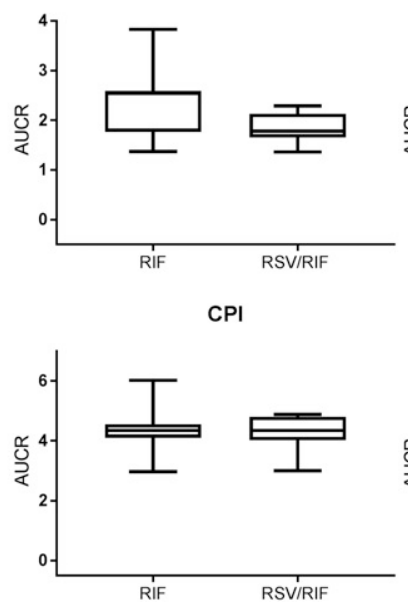

GDCA
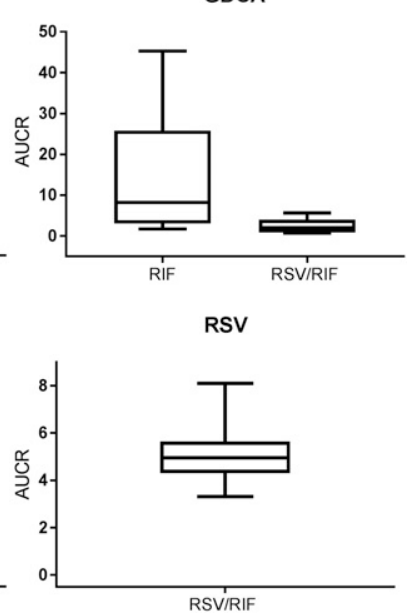

Fig. 2. Box and whisker plots of AUCRs for TDA, HDA, GCA, GDCA, TDCA, CPI, CPII, and rosuvastatin (RSV) estimated on two occasions in the presence of rifampicin (RIF) or RSV/RIF. Whiskers represent the $25 \%$ and $75 \%$ quartiles; ends of the whiskers show the minimum and maximum observed concentrations. The median AUCR is represented by a horizontal line within the box.

observed for CPI and the remaining biomarkers investigated (Supplemental Figs. 5 and 6). Only HDA showed a significant positive correlation between the rifampicin $C_{\max , \mathrm{u}}$ and biomarker AUCR (Fig. 4), whereas no significant trend was observed for other biomarkers (Supplemental Figs. 7 and 8). In addition to individual biomarkers, multivariate regression analysis was performed to assess whether information on the AUCR of CPI in combination with any of the selected biomarkers would capture the rosuvastatin AUCR. Since the AUCRs of CPI and CPIII were highly correlated, monitoring both biomarkers provided no improvement in correlation with the rosuvastatin AUCR. Monitoring of both CPI and GDCA showed a significant positive correlation with the rosuvastatin AUCR, but this correlation was weaker than for GDCA alone; similar trends were seen with TDCA (Supplemental Table 6). Overall, the multivariate analysis could not provide conclusive recommendations for the combination of biomarkers that would predict correctly the rosuvastatin interaction with rifampicin since the number of subjects available for paired analysis was small ( $n=7$ in most cases). Interestingly, monitoring of CPI, TDA, and rifampicin $C_{\max , \mathrm{u}}$ predicted correctly $90 \%$ of the rosuvastatin AUCRs $(P<0.05)$ (Supplemental Table 7); although this analysis focused solely on rifampicin, it highlighted the importance of consideration of perpetrator PK properties.

Stability of $\boldsymbol{T}_{\mathbf{0}}$ Plasma Concentrations. The mean $T_{0}$ plasma concentration for each of the 20 endogenous molecules investigated was calculated for each interaction occasion (details are given in Supplemental Table 4), and individual values for the selected subset are displayed in Fig. 5. CPI was used as a reference biomarker since it displayed the most stable baseline across the three occasions with low inter- and intraindividual variability ( $<25 \%$ ) (Barnett et al., 2018). Although there were no significant differences in $T_{0}$ biomarker plasma concentrations between the three occasions $(P>0.05)$, high inter- and intraindividual variabilities were evident, in particular for taurocholic acid (156\%). Similar to CPI, LCA and CPIII showed low (9\%-39\%) inter- and intraindividual variability in $T_{0}$ (Fig. 5; Supplemental Table 4). Despite stability in baseline $T_{0}$, LCA was not deemed suitable due to marginal transport via OATP1B estimated from rifampicin interaction data (Supplemental Tables 1 and 3). In the subset of biomarkers selected, the following rank order in terms of $T_{0}$ stability was noted: CPIII, HDA, TDA, GCA, GDCA, and TDCA. In contrast to CPIII, HDA, and TDA, high interindividual variability in $T_{0}$ was evident for GCA, GDCA, and TDCA (69\%-113\% across three occasions). TDCA displayed the highest interindividual variability in $T_{0}$ of $>100 \%$ in each occasion, which was also reflected in the intraindividual variability.

Stability of Biomarker Baseline Plasma Concentrations over 24 Hours. Baseline plasma concentration-time profiles over a 24-hour period for each of the selected endogenous biomarkers are shown in Supplemental Fig. 9. Each plasma concentration for a given time point throughout the duration of the rosuvastatin (control) phase was compared with the concentration at $T_{0}$ to determine any significant differences. CPIII was the only biomarker to show no significant deviations over 24 hours from the concentration measured at $T_{0}$, analogous to reference biomarker CPI. In the case of TDA, a significant increase in plasma concentration was observed between 3 and 8 hours into the study. A similar observation was also noted for HDA, where a significant increase in its plasma concentration was measured at 12 hours compared with $T_{0}$. In contrast, bile acids GCA, GDCA, and TDCA showed a significant decrease in plasma concentrations within the initial 4 hours. Although GCA, GDCA, and TDCA all showed an increase in plasma concentrations at 6 hours, this trend was not significant due to the high variability between subjects (Supplemental Fig. 10).

The baseline AUC of CPI was also compared with data from five recently reported clinical studies (Lai et al., 2016; Kunze et al., 2018; Liu et al., 2018; Shen et al., 2018; Takehara et al., 2018). Mean baseline AUCs were comparable irrespective of gender or ethnicity of subjects, with a weighted mean $\mathrm{AUC}_{0-24}$ of $18.9 \mathrm{nM} \cdot \mathrm{h}(n=91$, CV $24 \%$ ) (Table 2$) . S L C O 1 B 1 \mathrm{c} .521 \mathrm{~T}>\mathrm{C}$ genotype status for 91 subjects was 37 c.521 TT (wild type), three c.521 TC, whereas genotype data for 51 subjects were not reported (Table 2). Analogous analysis was done for the baseline AUCs for BAs (Supplemental Fig. 10), but in contrast to CPI more than half of the BAs investigated showed a difference of $>2$-fold in baseline AUC across studies $(n=$ 23 subjects in total, three studies). 
TDA

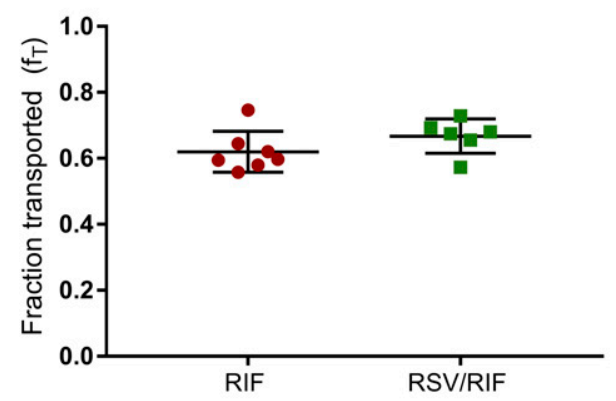

GCA

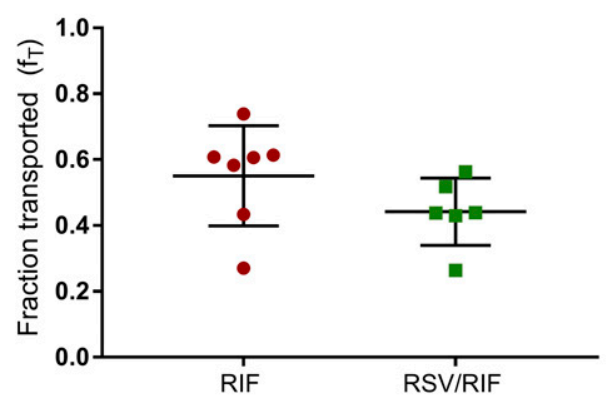

TDCA

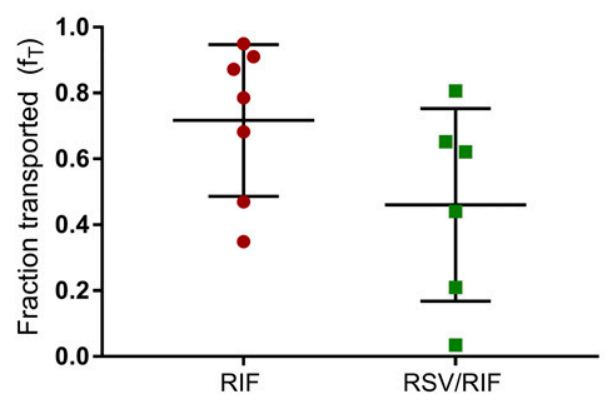

HDA

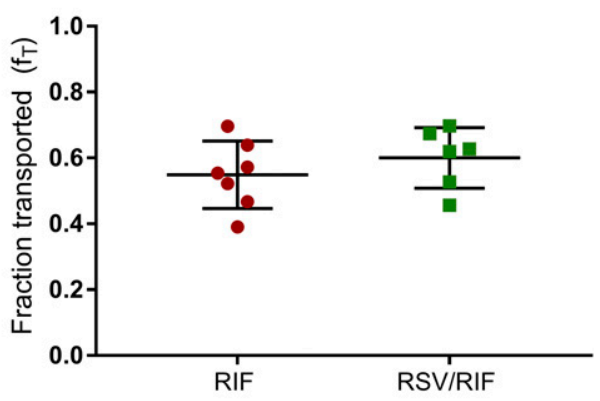

GDCA

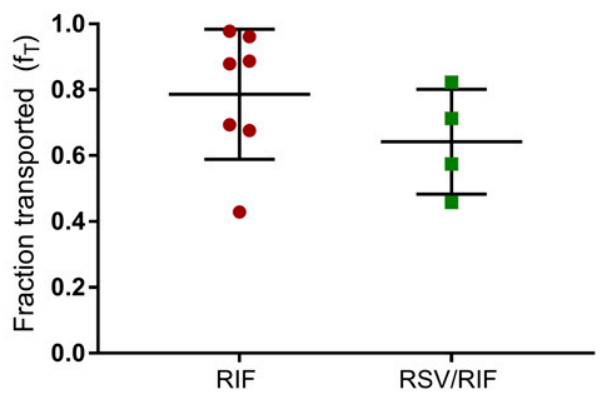

Fig. 3. Individual $f_{T}$ values calculated for TDA, HDA, GCA, GDCA, TDCA, and CPIII from two interaction occasions in the presence of rifampicin (RIF) or combined RSV/RIF. Error bars represent the S.D.
Reproducibility of EBI. The weighted mean AUCRs in the presence of rifampicin ranged from 2.1 to 8.5 (GCA and GDCA) for the selected biomarker subset. However, differences in the AUCR between inhibition occasions were evident, in particular for GDCA, where an AUCR of 13.7 was observed in the rifampicin occasion, compared with 2.5 in the rosuvastatin/rifampicin occasion (Fig. 6). Four out of the six biomarkers demonstrated a reproducible EBI with the AUCR of the biomarker within bioequivalence limits for both occasions. GDCA and TDCA were outliers, unable to reproduce the interaction in the rosuvastatin/rifampicin occasion. Additionally, a separate comparison of the AUCR of BAs between two studies in Japanese volunteers showed comparable magnitudes of EBI (Fig. 6). However, there were some exceptions, since the sulfate conjugate of glycochenodeoxycholic acid (GCDCA-S), chenodeoxycholic acid, and taurohyodeoxycholic acid/tauroursodeoxycholic acid showed differences in AUCR of $>2$-fold between studies (Fig. 6).

Effect of Rifampicin on Plasma Exposure of Endogenous Molecules. CPI was the most sensitive biomarker, since significant increases in CPI plasma concentration were observed after 30 minutes into the study following administration with rifampicin, reflecting the rifampicin PK profile (Supplemental Fig. 11; Supplemental Table 8). CPIII, TDA, and HDA also demonstrated sensitivity to perpetrator PK, since they showed significant increases in plasma exposure after 1 hour following administration of rifampicin. In contrast, the BAs (GCA, GDCA, and TDCA) showed a delayed increase in plasma exposure by rifampicin, which was only observed 4-6 hours into the study (Supplemental Fig. 11; Supplemental Table 8). Furthermore, significant increases in plasma concentrations of these biomarkers were observed after rifampicin had reached its $C_{\max }$ (rifampicin $T_{\max }$ is approximately 2.5 hours) (Lai et al., 2016). Given that a significant increase in BA plasma exposure is not observed until 6 hours after administration of rifampicin, the duration of the EBI study needs to be reevaluated for these biomarkers.

Subject $\mathrm{X}$ was used as a case example because of its altered plasma rifampicin systemic PK between two interaction occasions and delayed $T_{\max }$ (Fig. 7). These changes in rifampicin PK were reflected in altered plasma profiles of CPI, CPIII, TDA, and HDA (all showed delay in $T_{\max }$ compared with the rosuvastatin/rifampicin occasion), whereas no difference in profiles was observed between the occasions for GCA, GDCA, and 

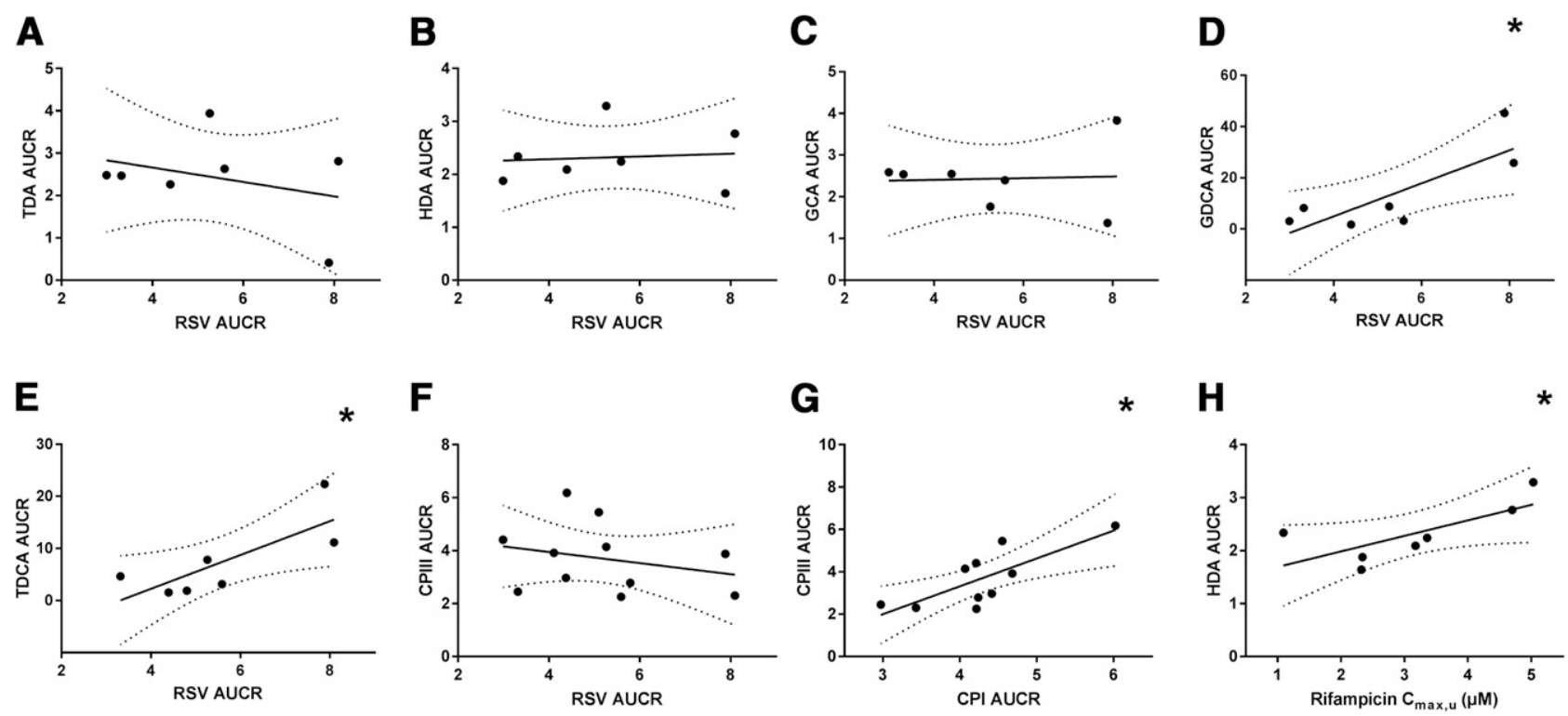

Fig. 4. Correlation between the endogenous biomarker AUCR with (A-F) rosuvastatin (RSV) AUCR $(n=6),(\mathrm{G})$ CPI AUCR $(n=10)$, and (H) the unbound maximum plasma concentration of rifampicin $\left(C_{\max , \mathrm{u}}\right)$ in the rifampicin interaction occasion $(n=7)$, obtained in the same individuals. Dashed lines represent the $95 \%$ confidence interval; * denotes a significant nonzero slope $(P<0.05)$.

TDCA (Fig. 7). In addition, subject $\mathrm{X}$ was used for the relative comparison of plasma concentration-time profiles of all selected biomarkers in the same subject (Fig. 7; data for additional subjects are shown in Supplemental Fig. 12). Overall, CPIII exhibited the lowest concentrations in the plasma followed by CPI, whereas GCA demonstrated the highest plasma concentrations followed by GDCA. HDA, TDA, and TDCA were generally in between these extremes with no clear trend in their rank order (Fig. 7; Supplemental Fig. 12). The subject X case illustrated the scenario where plasma influx of BAs occurred long after the $T_{\max }$ of the inhibitor, reducing the maximal effect of transporter inhibition. Timing of food intake with oral administration of the inhibitor may result in a greater interaction with the BAs, as demonstrated in Blank et al. (2018), where food was given within the first 30 minutes of the study.

\section{Discussion}

Availability of interaction data for 20 endogenous molecules, together with CPI and a clinical probe rosuvastatin in the same subjects, provided an excellent opportunity for cross comparison and evaluation of potential OATP1B biomarkers. So far, detailed evaluation of rifampicin AUCR and $\mathrm{f}_{\mathrm{T}}$, in addition to stability of biomarker $T_{0}$ and $\mathrm{AUC}_{0-24}$, was only investigated for CPI (Lai et al., 2016; Barnett et al., 2018). For the first time, an analogous approach was undertaken here for selected endogenous biomarkers (TDA, HDA, GCA, GDCA, TDCA, and CPIII) (Table 1) and their utility was evaluated against rosuvastatin and CPI (or in combination with CPI). CPI was considered as a reference OATP1B biomarker based on interaction data published thus far and low inter- and intraindividual variability in its baseline $T_{0}$ and plasma concentrations over 24 hours, with no evident postprandial effect (Lai et al., 2016; Barnett et al., 2018; Kunze et al., 2018; Shen et al., 2018). In the current analysis, minor fluctuations in 24-hour baseline were observed for TDA and HDA, suggesting a possible food effect. Nevertheless, significant increases in TDA and HDA plasma concentrations were observed within 1 hour of rifampicin administration. In contrast to CPI, TDA, and HDA, high variability and fluctuations in baseline concentrations were apparent for GCA, GDCA, and TDCA. Within the first 4 hours of the control phase, these BAs showed a significant decrease in plasma concentration compared with $T_{0}$, followed by an increase at 6 hours (the trend was not significant due to high interindividual variability) (Supplemental Fig. 9). Increases in baseline plasma BAs

TABLE 1

Summary of AUCR, $f_{\mathrm{T}}$, EBI reproducibility, and correlation with rosuvastatin magnitude of rifampicin interaction for selected biomarkers Numbers in parenthesis represent the range across both inhibition occasions.

\begin{tabular}{lcccc}
\hline Biomarker & Weighted Mean AUCR & Weighted Mean f & $\begin{array}{r}\text { Correlation with Rosuvastatin } \\
\text { AUCR }\end{array}$ & $\begin{array}{c}\text { Reproducibility of Rifampicin EBI } \\
\text { (AUCR within 1.25-fold) }\end{array}$ \\
\hline TDA & $2.8(2.3-3.9)$ & $0.64(0.56-0.74)$ & No & Yes \\
HDA & $2.4(1.6-3.3)$ & $0.57(0.39-0.69)$ & No & Yes \\
GCA & $2.1(1.4-3.8)$ & $0.5(0.27-0.74)$ & No & Yes \\
GDCA & $8.5(0.68-45)$ & $0.74(0.46-0.96)$ & Yes & No \\
TDCA & $5.1(1-22.3)$ & $0.59(0.04-0.95)$ & Yes & No \\
CPIII & $3.6(2.3-6)$ & $0.7(0.55-0.84)$ & No & Yes \\
CPI & $4.3(3-6)$ & $0.76(0.66-0.83)$ & No & \\
\hline
\end{tabular}

\footnotetext{
${ }^{a}$ The only biomarker that showed positive correlation with CPI AUCR.
} 
TDA

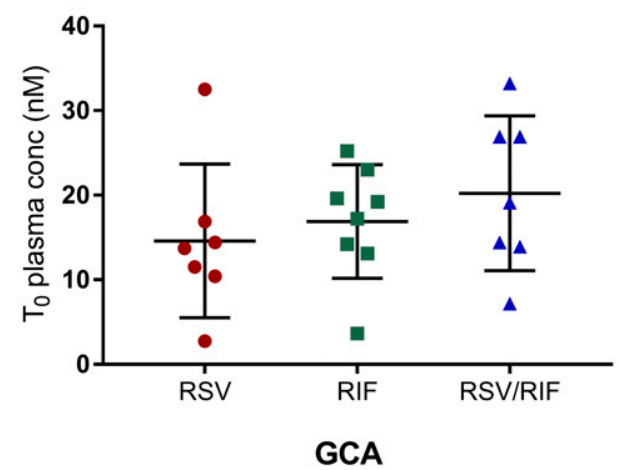

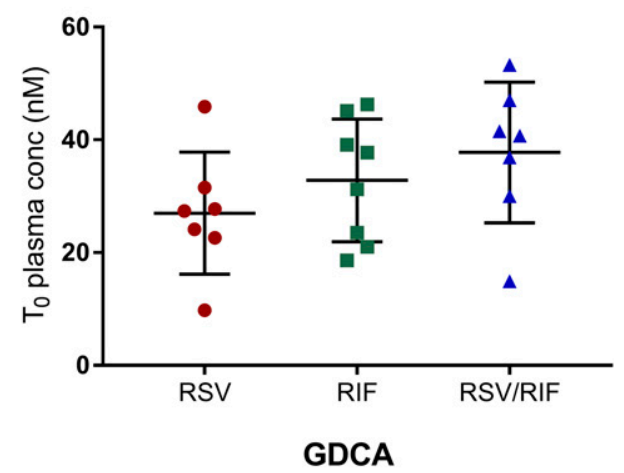
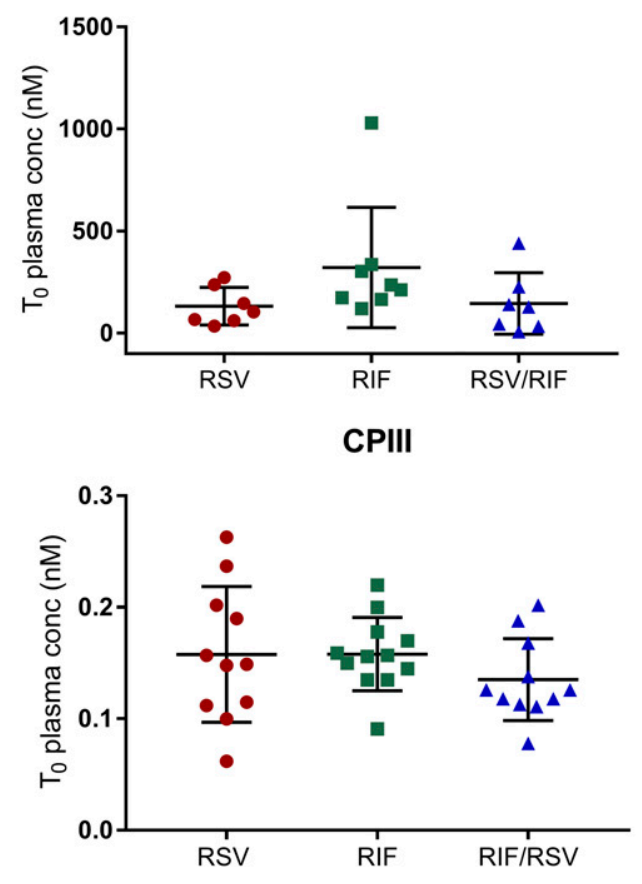

Fig. 5. Individual $T_{0}$ plasma concentrations for TDA, HDA, GCA, GDCA, TDCA, and CPIII measured on three occasions prior to the administration of rosuvastatin (RSV), rifampicin (RIF), or RSV/RIF. Error bars represent the S.D

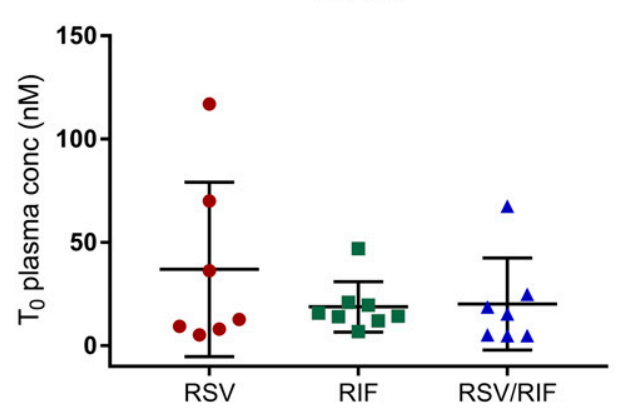

are most likely a result of their postprandial influx from the gastrointestinal tract rather than BA synthesis since only $5 \%$ of the total BA pool is synthesized daily (Trauner and Boyer, 2003). These fluctuations in GCA, GDCA, and TDCA plasma concentrations over the initial 4-6 hour period of the control phase highlight a need for careful clinical study design if BAs are to be used for monitoring of OATP1B activity in vivo. Findings from the current analysis are in agreement with the AUC data collated for BAs from different studies ( $n=23$ subjects) that showed $>2$-fold difference in baseline exposure of these biomarkers (Supplemental Fig. 10). In contrast, collated CPI baseline AUC data from 91 subjects from different ethnic groups were very comparable $(<25 \% \mathrm{CV})$ (Table 2). These data suggest that a reduced number of data points could be sufficient to define CPI baseline in a prospective EBI study, whereas this approach or reliance on biomarker AUC data from previous studies is not a feasible method when using BAs.

Synthesis of endogenous molecules is an important consideration in EBI clinical study design and data interpretation. Supplemental Table 9 highlights the rate-limiting steps in the synthesis of coproporphyrins, bile acids, and fatty acids and the factors that may affect regulation of these pathways through nuclear receptors. Additional consideration is a potential effect of transporter inhibitor on the synthesis of an endogenous biomarker, as discussed previously (Barnett et al., 2018). Multiple doses of rifampicin may affect synthesis of certain biomarkers (e.g., coproporhyrins and BAs) via pregnane $\mathrm{X}$ receptor activation. A single dose of rifampicin (used in OATP1B DDI studies) is not expected to have any effect and has only been reported to decrease plasma exposure of intermediate C4 in BA synthesis (Takehara et al., 2017). Given that daily synthesis contributes to a minor portion of circulating BAs, the impact on the total BA plasma concentration is likely to be minor. Kunze et al. (2018) reported a reduction in CPI AUCR following 5 days of rifampicin dosing compared with a single dose DDI. This finding is in contrast to the expected increase in CPI as a result of upregulation of haem synthesis associated with cytochrome P450 inducers such as rifampicin (Anderson et al., 2005). Potential OATP1B1 induction was ruled out since CPI $T_{0}$ was not affected by multiple rifampicin doses. Similarly, significant effects of autoinduction of rifampicin CYP3A4 metabolism were ruled out by the authors since even with the anticipated 1.5- to 2-fold decrease in rifampicin plasma concentration, these would still be significantly in excess of rifampicin 
TABLE 2

CPI baseline AUC and demographics of participants across several endogenous biomarker clinical interaction studies

\begin{tabular}{|c|c|c|c|c|c|c|c|c|c|}
\hline \multirow[b]{2}{*}{ Parameter } & \multicolumn{9}{|c|}{ Reference } \\
\hline & $\begin{array}{l}\text { Lai } \\
\text { et al. } \\
(2016)\end{array}$ & $\begin{array}{l}\text { Kunze } \\
\text { et al. } \\
(2018)\end{array}$ & $\begin{array}{l}\text { Takehara } \\
\text { et al. } \\
(2018)\end{array}$ & $\begin{array}{l}\text { Liu } \\
\text { et al. } \\
\text { (2018) }\end{array}$ & & & & Shen et al. (2018) & \\
\hline Sex & Male & Female & Male & Female & Male & Male & Male & Male & Male \\
\hline Age & $18-45$ & NR & $26-36$ & NR & $26-47$ & $26-47$ & $26-47$ & $26-47$ & $26-47$ \\
\hline Ethnicity & Indian & NR & Japanese & NR & Black & White & Hispanic & $\begin{array}{c}\text { Black (8), White (10), } \\
\text { Hispanic (9), American } \\
\text { Indian (1) }\end{array}$ & $\begin{array}{c}\text { Black (8), White (10), } \\
\text { Hispanic (9), American } \\
\text { Indian (1) }\end{array}$ \\
\hline $\begin{array}{l}S C L O 1 B 1 \text { c.521 } \\
\mathrm{T}>\mathrm{C} \text { status }\end{array}$ & WT & NR & NR & NR & NR & NR & NR & $\begin{array}{c}3 \text { c.521 TC, } 25 \text { c.521 TT } \\
\text { (WT) }\end{array}$ & $\begin{array}{c}3 \text { c.521 TC, } 25 \text { c.521 TT } \\
\text { (WT) }\end{array}$ \\
\hline Number of subjects & 12 & 12 & 8 & 15 & 4 & 3 & 9 & 14 & 14 \\
\hline $\begin{array}{l}\text { Mean CPI baseline } \\
\operatorname{AUC}_{(0-24)}(\mathrm{nM} \cdot \mathrm{h})\end{array}$ & 20.9 & $14.81^{a}$ & 16.1 & 24.23 & 21.08 & 17.49 & 15.32 & 16.88 & 20.69 \\
\hline
\end{tabular}

NR, not reported; OATP1B1 WT, wild type.

${ }^{a}$ Extrapolated from the 6-hour study.

OATP1B IC 50 (0.13 $\mu \mathrm{M}$, Barnett et al., 2018). It is important to note that in Kunze et al. (2018) study rifampicin was coadministered with an in-house victim drug, which may have also had an effect on CPI plasma exposure. All of the aforementioned results highlight the requirement for additional data to improve our understanding of factors that alter synthesis and exposure of endogenous biomarkers. In addition to synthesis, the impact of disease, genetic polymorphism of transporters of interest, postprandial effect, and perpetrator PK need to be considered in the EBI study design/data interpretation. The subject $\mathrm{X}$ case and altered rifampicin $\mathrm{PK}$ between the two inhibition occasions were captured by CPI, CPIII, HDA, and TDA and changes in their plasma concentrations, in contrast to profiles for GCA, GDCA, and TDCA (Fig. 7). Additionally, these BAs showed no significant increases in plasma concentrations compared with $T_{0}$ until $>4$ hours after rifampicin administration (Supplemental Fig. 11; Supplemental Table 8), suggesting that BA response to OATP inhibition was most likely concealed by food effect (the trend evident in all subjects). This sensitivity to alterations in the perpetrator plasma $\mathrm{PK}$ is an important consideration when EBI data are used for estimation of in vivo OATP1B $K_{\mathrm{i}}$ by

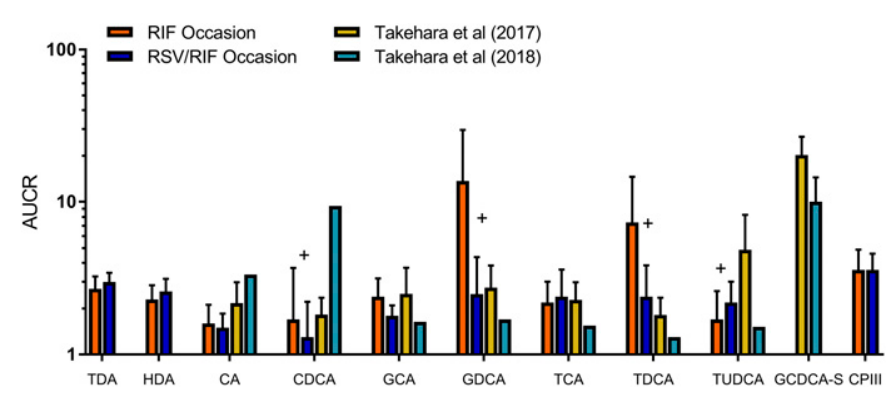

Fig. 6. Reproducibility of EBI magnitude following a single $600 \mathrm{mg}$ dose of rifampicin in the same Indian individuals on two different inhibition occasions [rifampicin (RIF) or rosuvastatin (RSV) with RIF (RSV/RIF)] and two different groups of Japanese volunteers reported in Takehara et al. (2017, 2018). Error bars represent the S.D.; + denotes an AUCR of $>1.25$-fold difference between occasions in the same individuals. Tauroursodeoxycholic acid (TUDCA) represents combined taurohyodeoxycholic acid/TUDCA in current analysis, but TUDCA only in the data from the Takehara et al. $(2017,2018)$ studies. Glycochenodeoxycholic acid (GCDCA)/GDCA and taurochenodeoxycholic acid/TDCA in Takehara et al. $(2017,2018)$ were measured together and are represented on the chart by GDCA and TDCA, respectively. modeling approaches, as was done for CPI-rifampicin (Barnett et al., 2018). Ideally, inhibitor PK and biomarker interaction data from the same subjects should be employed. Use of simulated population average inhibitor PK data as an alternative (Yoshida et al., 2018) may result in substantial differences in the estimated transporter $K_{\mathrm{i}}$, if the simulated PK differs from perpetrator concentrations in the EBI study driving the observed changes in biomarker exposure.

Chu et al. $(2017,2018)$ highlighted the importance of reproducible EBIs for the validation of biomarkers as transporter probes. In addition to CPI, this study showed the reproducibility of CPIII, TDA, and HDA interactions with rifampicin, with AUCRs between occasions within bioequivalence limits (Fig. 6); opposite trends were noted for GDCA and TDCA ( $>2$-fold difference in the AUCR). An important consideration is the assumption made here that rosuvastatin had no impact on the PK of the endogenous molecules investigated. No effect of rosuvastatin on CPI, CPIII, HDA, and TDA PK supports this assumption. However, in the case of BAs, differences in the magnitude of EBI were evident between the rifampicin-only and rosuvastatin/rifampicin occasions (Figs. 2, 6, and 7), which may have also affected the assessment of reproducibility of EBIs for these biomarkers. Recently, GCDCA-S has been highlighted as a promising OATP1B endogenous biomarker (Yee et al., 2016; Takehara et al., 2017, 2018), with a mean 10 -fold increase in AUC after administration of a $600 \mathrm{mg}$ oral dose of rifampicin. Although a strong correlation between the AUC of GCDCA-S and the AUC of clinical probe atorvastatin was reported, the relationship between the fold change in atorvastatin and GCDCA-S AUC across rifampicin dose range was not investigated. In contrast, a previous study by the same group resulted in a much higher mean GCDCA-S AUCR of 20 -fold (600 mg rifampicin, single dose), despite similar demographics (Takehara et al., 2017, 2018), confirming further variability challenges noted for BAs and corresponding metabolites between occasions and individuals (Fig. 6).

Overlapping substrate specificity is a common problem for both drug and endogenous transporter probes (Chu et al., 2018). Out of the biomarkers selected for investigation in this study, none are entirely selective for OATP1B1, including CPI; transporters involved in their hepatic uptake and efflux collated from in vitro and clinical data are listed in Supplemental Table 10. In addition to OATP1B1, TDA and HDA are also transported by OAT1 and OAT3 (Yee et al., 2016), which 
A

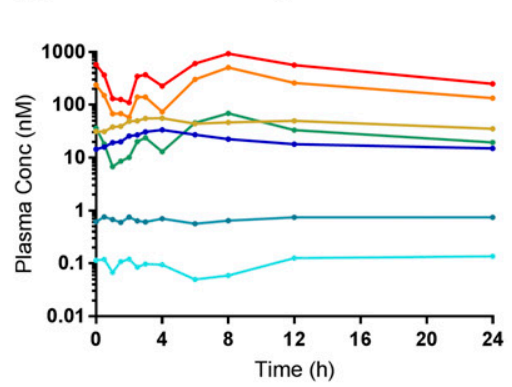

D

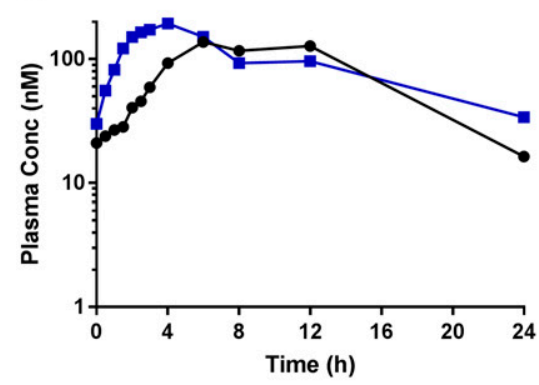

G

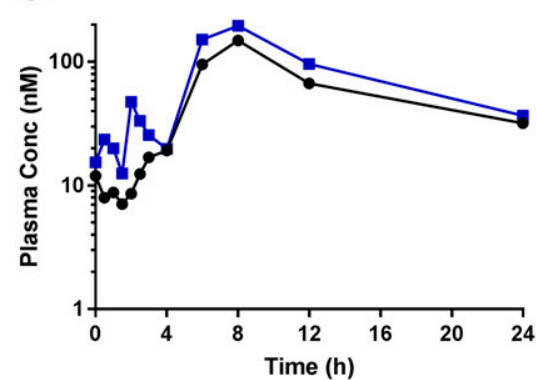

C

TDA
B

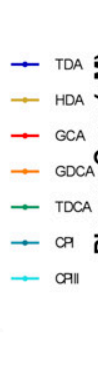

E

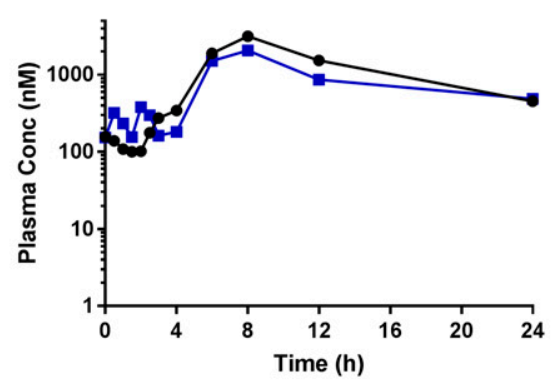

H
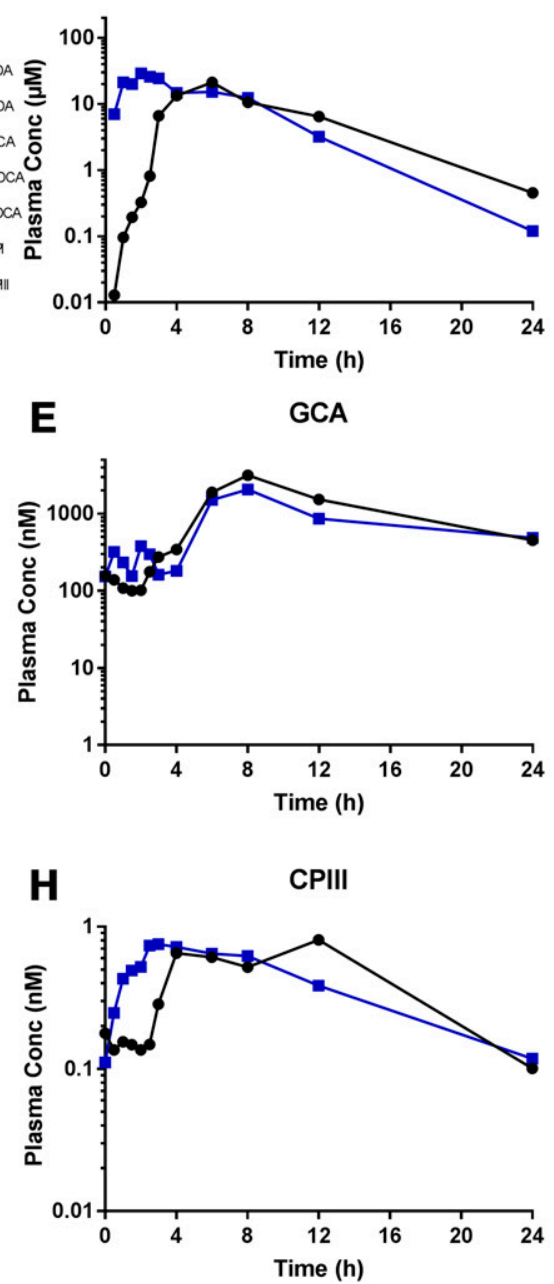

GCA

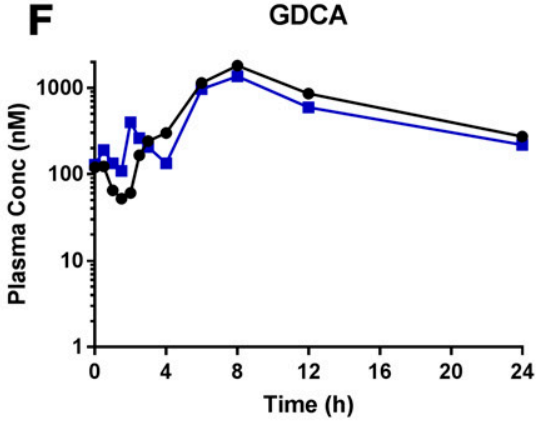

I

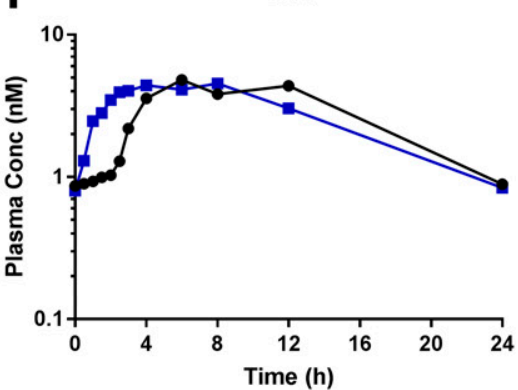

Fig. 7. (A) Plasma concentration-time profiles for selected biomarkers in subject X under baseline conditions (rosuvastatin). (B-I) Plasma concentration-time profiles of rifampicin, TDA, HDA, GCA, GDCA, TDCA, CPIII, and CPI in subject X in the presence of rifampicin (black line) and rosuvastatin/rifampicin (blue line).

may complicate differentiation of the transporter interactions in the liver and kidney (unless supported by renal clearance data for the biomarker). In the case of CPI, hepatic uptake is driven by OATP1B1 and 1B3 (Bednarczyk and Boiselle, 2016; Shen et al., 2017; Kunze et al., 2018), but involvement of multidrug resistance proteins (MPR) 2 and 3 in its efflux may have implications when investigating dual OATP1B/MRP inhibitors (Gilibili et al., 2017; Kunze et al., 2018) or in patient populations because of reported disease-associated changes in transporter activity/interplay (Evers et al., 2018).

Multivariate regression analysis was performed here to assess whether the combination of selected biomarkers would recapitulate correctly the DDI risk observed with a clinical probe rosuvastatin. Consideration of both CPI and CPIII AUCR data did not improve the correlation with rosuvastatin AUCR since these biomarkers are highly correlated with each other (Fig. 4), indicating that monitoring of CPIII provides no additional benefit in understanding OATP1B-mediated DDI risk to CPI. Encouraging trends were seen with a combination of GDCA/TDCA with CPI AUCR, but further studies are required to determine if these biomarkers are beneficial for the assessment of dual OATP1B/NTCP inhibitors, considering the recently reported substantial increase in BA AUCs in the presence of the potent sodium-NTCP inhibitor Myrcludex ( $f_{\mathrm{T}}$ estimate $>0.95$ ) (Blank et al., 2018). Overall, the small number of subjects available for the multivariate analysis prevents any conclusive recommendation for the best combination of biomarkers to reflect the OATP1B-mediated DDI risk with rosuvastatin. In addition to the requirement for data sets from larger cohorts, this multivariate evaluation needs to be expanded against other OATP1B clinical probes (e.g., pitavastatin and atorvastatin) and other perpetrators than rifampicin.

In conclusion, systematic evaluation of AUCR, $\mathrm{f}_{\mathrm{T}}, T_{0}$, and $\mathrm{AUC}_{0-24}$ stability was performed for 20 potential endogenous biomarkers of OATP1B. CPIII was the only biomarker that showed a direct relationship to the magnitude of rifampicin interaction observed for CPI. Despite its robustness, CPIII demonstrated no additional benefit over CPI for evaluating OATP1B DDI risk. CPI data from 91 subjects showed minimal interindividual variability and high EBI reproducibility, confirming its superiority as an OATP1B endogenous biomarker. Further evaluation of HDA and TDA against a panel of OATP1B inhibitors is required to confirm their utility (alone and in combination with CPI). GDCA and 
TDCA AUCRs showed a significant positive correlation to the magnitude of rosuvastatin DDI with rifampicin in the same individuals, but no correlation to CPI interaction. The lack of AUCR reproducibility and large interindividual variability in the 24 -hour baseline, confounded by the postprandial effect, needs to be considered when using BAs for evaluation of OATP1B DDIs. The benefits of monitoring combined biomarkers (CPI, one bile acid, and one fatty acid) need to be confirmed with larger data sets and against other OATP1B clinical probes and perpetrators.

\section{Authorship Contributions}

Participated in research design: Barnett, Shen, Humphreys, Galetin.

Performed data analysis: Barnett, Ogungbenro, Galetin.

Wrote or contributed to the writing of the manuscript: Barnett, Ogungbenro, Ménochet, Shen, Humphreys, Galetin.

\section{References}

Anderson KE, Bloomer JR, Bonkovsky HL, Kushner JP, Pierach CA, Pimstone NR, and Desnick RJ (2005) Recommendations for the diagnosis and treatment of the acute porphyrias. Ann Intern Med 142:439-450.

Barnett S, Ogungbenro K, Ménochet K, Shen H, Lai Y, Humphreys WG, and Galetin A (2018) Gaining mechanistic insight into coproporphyrin I as endogenous biomarker for OATP1B-mediated drug-drug interactions using population pharmacokinetic modeling and simulation. Clin Pharmacol Ther 104:564-574.

Bednarczyk D and Boiselle C (2016) Organic anion transporting polypeptide (OATP)-mediated transport of coproporphyrins I and III. Xenobiotica 46: 457-466.

Blank A, Eidam A, Haag M, Hohmann N, Burhenne J, Schwab M, van de Graaf S, Meyer MR, Maurer HH, Meier K, et al. (2018) The NTCP-inhibitor myrcludex B: effects on bile acid disposition and tenofovir pharmacokinetics. Clin Pharmacol Ther 103:341-348.

Chu X, Chan GH, and Evers R (2017) Identification of endogenous biomarkers to predict the propensity of drug candidates to cause hepatic or renal transportermediated drug-drug interactions. J Pharm Sci 106:2357-2367.

Chu X, Liao M, Shen H, Yoshida K, Zur AA, Arya V, Galetin A, Giacomini KM, Hanna I, Kusuhara H, Lai Y, Rodrigues AD, Sugiyama Y, Zamek-Gliszczynski MJ, and Zhang L; International Transporter C (2018) Clinical Probe Drugs and Endogenous Biomarkers as Substrates for Transporter-Related Drug-Drug Interaction Evaluation: Perspectives from the International Transporter Consortium. Clin Pharmacol Ther 104(5):836-864.

Chu X, Shih SJ, Shaw R, Hentze H, Chan GH, Owens K, Wang S, Cai X, Newton D, Castro-Perez J, et al. (2015) Evaluation of cynomolgus monkeys for the identification of endogenous biomarkers for hepatic transporter inhibition and as a translatable model to predict pharmacokinetic interactions with statins in humans. Drug Metab Dispos 43:851-863.

Dawson PA (2011) Role of the intestinal bile acid transporters in bile acid and drug disposition. Handb Exp Pharmacol 201:169-203.

Evers R, Piquette-Miller M, Polli JW, Russel FGM, Sprowl JA, Tohyama K, Ware JA, de Wildt SN, Xie W, Brouwer KLR; International Transporter Consortium (2018) Disease-associated changes in drug transporters may impact the pharmacokinetics and/or toxicity of drugs: a white paper from the International Transporter Consortium. Clin Pharmacol Ther 104(5):900-915.

Food and Drug Administration (2017) Guidance for industry, in vitro metabolism and transporter mediated drug-drug interaction studies. https://www.fda.gov/downloads/Drugs/Guidances/UCM581965.pdf

Galetin A, Zhao P, and Huang SM (2017) Physiologically based pharmacokinetic modeling of drug transporters to facilitate individualized dose prediction. J Pharm Sci 106:2204-2208.

Gertz M, Cartwright CM, Hobbs MJ, Kenworthy KE, Rowland M, Houston JB, and Galetin A (2013) Cyclosporine inhibition of hepatic and intestinal CYP3A4 uptake and efflux transporters: application of PBPK modeling in the assessment of drug-drug interaction potential. Pharm Res 30:761-780.

Giacomini KM, Huang SM, Tweedie DJ, Benet LZ, Brouwer KL, Chu X, Dahlin A, Evers R, Fischer V, Hillgren KM, et al.; International Transporter Consortium (2010) Membrane transporters in drug development. Nat Rev Drug Discov 9: $215-236$

Gilibili RR, Chatterjee S, Bagul P, Mosure KW, Murali BV, Mariappan TT, Mandlekar S, and Lai Y (2017) Coproporphyrin-I: a fluorescent, endogenous optimal probe substrate for ABCC2 (MRP2) suitable for vesicle-based MRP2 inhibition assay. Drug Metab Dispos 45:604-611.

Guo Y, Chu X, Parrott NJ, Brouwer KLR, Hsu V, Nagar S, Matsson P, Sharma P, Snoeys J, Sugiyama Y, et al.; International Transporter Consortium (2018) Advancing predictions of tissue and intracellular drug concentrations using in vitro, imaging and physiologically based pharmacokinetic modeling approaches. Clin Pharmacol Ther 104(5):865-889.

Hegade VS, Jones DE, and Hirschfield GM (2017) Apical sodium-dependent transporter inhibitors in primary biliary cholangitis and primary sclerosing cholangitis. Dig Dis 35:267-274.

Hibma JE, Zur AA, Castro RA, Wittwer MB, Keizer RJ, Yee SW, Goswami S, Stocker SL, Zhang X, Huang Y, et al. (2016) The effect of famotidine, a MATE1-selective inhibitor, on the pharmacokinetics and pharmacodynamics of metformin. Clin Pharmacokinet 55:711-721.

Jones HM, Chen Y, Gibson C, Heimbach T, Parrott N, Peters SA, Snoeys J, Upreti VV, Zheng M, and Hall SD (2015) Physiologically based pharmacokinetic modeling in drug discovery and development: a pharmaceutical industry perspective. Clin Pharmacol Ther 97:247-262.

Kunze A, Ediage EN, Dillen L, Monshouwer M, and Snoeys J (2018) Clinical investigation of coproporphyrins as sensitive biomarkers to predict mild to strong OATP1B-mediated drug-drug interactions. Clin Pharmacokinet DOI: 10.1007/ s40262-018-0648-3 [published ahead of print].

Lai Y, Mandlekar S, Shen H, Holenarsipur VK, Langish R, Rajanna P, Murugesan S, Gaud N, Selvam S, Date O, et al. (2016) Coproporphyrins in plasma and urine can be appropriate clinical biomarkers to recapitulate drug-drug interactions mediated by organic anion transporting polypeptide inhibition. J Pharmacol Exp Ther 358: 397-404.

Liu L, Cheeti S, Yoshida K, Choo E, Chen E, Chen B, Gates M, Singel S, Morley R, Ware J, et al. (2018) Effect of OATP1B1/1B3 inhibitor GDC-0810 on the pharmacokinetics of pravastatin and coproporphyrin I/III in healthy female subjects. J Clin Pharmacol 58:1427-1435.

Mok HY, Von Bergmann K, and Grundy SM (1977) Regulation of pool size of bile acids in man. Gastroenterology 73:684-690.

Müller F, Sharma A, König J, and Fromm MF (2018) Biomarkers for in vivo assessment of transporter function. Pharmacol Rev 70:246-277.

Prueksaritanont T, Chu X, Evers R, Klopfer SO, Caro L, Kothare PA, Dempsey C, Rasmussen S, Houle R, Chan G, et al. (2014) Pitavastatin is a more sensitive and selective organic anion-transporting polypeptide $1 \mathrm{~B}$ clinical probe than rosuvastatin. Br J Clin Pharmacol 78:587-598.

Rodrigues AD, Taskar KS, Kusuhara H, and Sugiyama Y (2018) Endogenous probes for drug transporters: balancing vision with reality. Clin Pharmacol Ther 103: $434-448$.

Shen H, Chen W, Drexler DM, Mandlekar S, Holenarsipur VK, Shields EE, Langish R, Sidik K, Gan J, Humphreys WG, et al. (2017) Comparative evaluation of plasma bile acids, dehydroepiandrosterone sulfate, hexadecanedioate, and tetradecanedioate with coproporphyrins I and III as markers of OATP inhibition in healthy subjects. Drug Metab Dispos 45:908-919.

Shen H, Christopher L, Lai Y, Gong J, Kandoussi H, Garonzik S, Perera V, Garimella T, and Humphreys WG (2018) Further studies to support the use of coproporphyrin I and III as novel clinical biomarkers for evaluating the potential for organic anion transporting polypeptide 1B1 and OATP1B3 inhibition. Drug Metab Dispos 46: 1075-1082.

Shen H, Dai J, Liu T, Cheng Y, Chen W, Freeden C, Zhang Y, Humphreys WG, Marathe P, and Lai Y (2016) Coproporphyrins I and III as functional markers of OATP1B activity: in vitro and in vivo evaluation in preclinical species. $J$ Pharmacol Exp Ther 357:382-393.

Snoeys J, Beumont M, Monshouwer M, and Ouwerkerk-Mahadevan S (2016) Mechanistic understanding of the nonlinear pharmacokinetics and intersubject variability of simeprevir: a PBPK-guided drug development approach. Clin Pharmacol Ther 99:224-234

Takehara I, Terashima H, Nakayama T, Yoshikado T, Yoshida M, Furihata K, Watanabe N, Maeda K, Ando O, Sugiyama Y, et al. (2017) Investigation of glycochenodeoxycholate sulfate and chenodeoxycholate glucuronide as surrogate endogenous probes for drug interaction studies of OATP1B1 and OATP1B3 in healthy Japanese volunteers. Pharm Res 34:1601-1614.

Takehara I, Yoshikado T, Ishigame K, Mori D, Furihata KI, Watanabe N, Ando O, Maeda K, Sugiyama Y, and Kusuhara H (2018) Comparative study of the dosedependence of OATP1B inhibition by rifampicin using probe drugs and endogenous substrates in healthy volunteers. Pharm Res 35(7):138.

Thakare R, Gao H, Kosa RE, Bi YA, Varma MVS, Cerny MA, Sharma R, Kuhn M, Huang B, Liu Y, et al. (2017) Leveraging of rifampicin-dosed cynomolgus monkeys to identify bile acid 3-O-sulfate conjugates as potential novel biomarkers for organic anion-transporting polypeptides. Drug Metab Dispos 45:721-733.

Trauner M and Boyer JL (2003) Bile salt transporters: molecular characterization, function, and regulation. Physiol Rev 83:633-671.

Tsamandouras N, Dickinson G, Guo Y, Hall S, Rostami-Hodjegan A, Galetin A, and Aarons L (2015) Development and application of a mechanistic pharmacokinetic model for simvastatin and its active metabolite simvastatin acid using an integrated population PBPK approach. Pharm Res 32:1864-1883.

Vaidyanathan J, Yoshida K, Arya V, and Zhang L (2016) Comparing various in vitro prediction criteria to assess the potential of a new molecular entity to inhibit organic anion transporting polypeptide 1B1. J Clin Pharmacol 56 (Suppl 7): S59-S72

Watanabe M, Watanabe T, Yabuki M, and Tamai I (2015) Dehydroepiandrosterone sulfate, a useful endogenous probe for evaluation of drug-drug interaction on hepatic organic anion transporting polypeptide (OATP) in cynomolgus monkeys. Drug Metab Pharmacokinet 30:198-204.

Yee SW, Giacomini MM, Hsueh CH, Weitz D, Liang X, Goswami S, Kinchen JM, Coelho A, Zur AA, Mertsch K, et al. (2016) Metabolomic and genome-wide association studies reveal potential endogenous biomarkers for OATP1B1. Clin Pharmacol Ther 100:524-536.

Yoshida K, Guo C, and Sane R (2018) Quantitative prediction of OATP-mediated drug-drug interactions with model-based analysis of endogenous biomarker kinetics. CPT Pharmacometrics Syst Pharmacol 7:517-524.

Address correspondence to: Dr. Aleksandra Galetin, Centre for Applied Pharmacokinetic Research, School of Health Sciences, University of Manchester, Stopford Building, Oxford Road, Manchester M13 9PT, UK. E-mail: Aleksandra.Galetin@manchester.ac.uk 